\title{
An Efficient Characteristic Method for the Magnetic Induction Equation with Various Resistivity Scales
}

\author{
Jiangguo (James) Liu \\ Department of Mathematics, Colorado State University, Fort Collins, CO 80523, USA \\ liu@math.colostate.edu \\ http://www.math.colostate.edu/ liu/
}

\begin{abstract}
In this paper, we develop an efficient characteristic finite element method (FEM) for solving the magnetic induction equation in magnetohydrodynamics (MHD). We carry out numerical experiments on a two dimensional test case to investigate the influence of resistivity at different scales. In particular, our numerical results exhibit how the topological structure and energy of the magnetic field evolve for different scales of resistivity. Magnetic reconnection can also be observed in the numerical experiments.
\end{abstract}

Keywords: characteristic method, convection-diffusion, magnetohydrodynamics(MHD), magnetic resistivity, numerical simulation.

\section{Introduction}

Magnetohydrodynamics (MHD) is the study of the interaction between the magnetic field and the flow of electrically conducting fluids, typically plasmas, liquid metals, or brine. The MHD equations are a combination of the Navier-Stokes equations for fluid motion and the Maxwell equations for electromagnetism coupled through Lorentz body force and Ohmic heating [2]3]:

$$
\begin{aligned}
\partial_{t} \rho+\nabla \cdot(\rho \mathbf{v})=0 & \text { (Mass conservation) } \\
\partial_{t}(\rho \mathbf{v})+\nabla \cdot(\rho \mathbf{v} \otimes \mathbf{v}-\mathbf{B} \otimes \mathbf{B})+\nabla p^{*}=\mathbf{0} & \text { (Momentum conservation) } \\
\partial_{t} E+\nabla \cdot\left[\left(E+p^{*}\right) \mathbf{v}-(\mathbf{v} \cdot \mathbf{B}) \mathbf{B}\right]=\varepsilon|\nabla \times \mathbf{B}|^{2} & \text { (Energy conservation) } \\
\partial_{t} \mathbf{B}=\nabla \times(\mathbf{v} \times \mathbf{B})+\varepsilon \Delta \mathbf{B} & \text { (Magnetic induction equation) } \\
\nabla \cdot \mathbf{B}=0, \quad \text { for all } t \geq 0 & \text { (Solenoidal property) }
\end{aligned}
$$

where $\rho$ is the mass density (per unit volume of the liquid), $\mathbf{m}=\rho \mathbf{v}$ the momentum density, $\mathbf{v}$ the velocity field, $\mathbf{B}$ the magnetic field, $E$ the total energy density, $\varepsilon$ the magnetic resistivity that represents the effect of Ohmic heating, $t$ the time variable, and $\otimes$ stands for the tensor product. 
Some estimates suggest that up to $99 \%$ of the entire universe is filled by plasmas [2. Man-made and natural plasmas can be found in plasma TVs, plasma lamps, lightning, aurora, and solar winds (plasmas flowing out from the Sun at a very high speed and in the radially outward direction). Table1 1 lists the resistivity of various types of plasmas. Among them, the $5 \mathrm{k} \mathrm{eV}$ plasma is used in large lab fusion experiments [2].

Table 1. The resistivity of various types of plasmas

\begin{tabular}{||c|c||c|c||}
\hline Plasma & Resistivity (ohm m) & Plasma & Resistivity (ohm m) \\
\hline Earth's ionosphere & $2 \times 10^{-2}$ & $100 \mathrm{eV}$ plasma & $5 \times 10^{-7}$ \\
Solar corona & $5 \times 10^{-5}$ & $5 \mathrm{k} \mathrm{eV}$ plasma & $1 \times 10^{-9}$ \\
Interstellar gas & $5 \times 10^{-7}$ & & \\
\hline
\end{tabular}

The simplest MHD is the ideal MHD model that assumes the fluid has no resistivity and can be treated as a perfect conductor. The ideal MHD equations can be reformulated as a hyperbolic system and then hyperbolic solvers apply. If the resistivity terms are retained, the model is referred as resistive MHD.

Ideal MHD is applicable for a limited time before resistivity becomes too important to ignore. This limited time span varies. It could be hundreds to thousands of years for the Sun, and hence much longer than the actual lifetime of a sunspot, so it is reasonable to ignore the resistivity. However, a meter-sized volume of seawater has a magnetic diffusion time measured in milliseconds. Instabilities existing in a plasma can increase its effective resistivity. The enhanced resistivity usually results from the formation of small scale structures such as current sheets or fine scale magnetic turbulence. In these situations, the ideal MHD model is broken. When resistivity is present, magnetic reconnection may happen, in which a plasma releases stored magnetic energy as waves, bulk mechanical acceleration of material, particle acceleration, or heat. In addition, most numerical methods for ideal MHD inevitably introduce numerical diffusion and finally break down. Therefore, efficient numerical methods for resistive MHD are much needed.

However, resistive MHD presents a challenge due to the widespread time scales, the nonlinear coupling, the fineness of spatial grids for resolving current sheets, etc. To tackle this multi-physics and multi-scale problem, one could adopt an operator decomposition approach. Efficient solvers based on characteristic tracking for MHD kinematics (the influence of the velocity field on the magnetic field) and the fluid equations (the first three equations in (1) can be solved as a hyperbolic system) are developed respectively. Strategies for controlling errors of coupling between the characteristic solvers and the hyperbolic solvers (reflection of the interaction of fluid motion and electromagnetism) are also to be investigated. 


\section{A Characteristic Finite Element Method for the Magnetic Field Induction Equation}

The magnetic induction equation and the solenoidal (divergence-free) property together describe the kinematics of MHD flows, i.e., the influence of the velocity field on the magnetic field. The divergence-free property $\nabla \cdot \mathbf{B}=0$ should be preserved by numerical methods, globally or locally, in the classical or weak sense. One approach is to express the magnetic field as the curl of a vector potential $\mathbf{A}$, i.e., to define $\mathbf{B}=\nabla \times \mathbf{A}$. This approach is particularly convenient in two dimensions, where $\mathbf{A}=\left(0,0, A_{3}\right)$ and $\mathbf{B}=\left(B_{1}, B_{2}, 0\right)=\left(\partial_{y} A_{3},-\partial_{x} A_{3}, 0\right)$. If the fluid is incompressible $(\nabla \cdot \mathbf{v}=0)$, then the magnetic field equation reduces to a scalar convection-diffusion equation about the magnetic potential $A_{3}$ :

$$
\partial_{t} A_{3}+\nabla \cdot\left(\mathbf{v} A_{3}-\varepsilon \nabla A_{3}\right)=0
$$

Convection-diffusion problems like equation (2) arise also in groundwater contaminant remediation, petroleum reservoir simulation, and many other applications. A general form for this type of problems is

$$
\left\{\begin{array}{l}
u_{t}+\nabla \cdot(\mathbf{v} u-\mathbf{D} \nabla u)=f(\mathbf{x}, t), \quad \mathbf{x} \in \Omega, t \in(0, T], \\
u(\mathbf{x}, 0)=u_{0}(\mathbf{x}), \quad \mathbf{x} \in \Omega, \\
\text { Suitable boundary conditions, }
\end{array}\right.
$$

where $\Omega \subset \mathbb{R}^{d}(d=1,2,3)$ is a domain with boundary $\Gamma=\partial \Omega, u(\mathbf{x}, t)$ is the unknown function, $\mathbf{v}(\mathbf{x}, t)$ a prescribed velocity field, $\mathbf{D}(\mathbf{x}, t)$ a diffusion matrix, and $f(\mathbf{x}, t)$ a source/sink term. Let $\mathbf{n}$ be the unit outward normal vector on $\Gamma$. We define the inflow, outflow, and noflow boundaries $\Gamma^{I}, \Gamma^{O}, \Gamma^{N}$ as

$\Gamma^{I}=\{\mathbf{x} \in \Gamma: \mathbf{v} \cdot \mathbf{n}<0\}, \quad \Gamma^{O}=\{\mathbf{x} \in \Gamma: \mathbf{v} \cdot \mathbf{n}>0\}, \quad \Gamma^{N}=\{\mathbf{x} \in \Gamma, \mathbf{v} \cdot \mathbf{n}=0\}$.

Dirichlet, Neumann, or Robin boundary conditions are posed as

$$
\begin{aligned}
& u(\mathbf{x}, t)=g_{1}^{\text {type }}(\mathbf{x}, t), \quad(\mathbf{x}, t) \in \Gamma^{\text {type }} \quad \text { (Dirichlet) }, \\
& -\mathbf{D} \nabla u(\mathbf{x}, t) \cdot \mathbf{n}=g_{2}^{\text {type }}(\mathbf{x}, t), \quad(\mathbf{x}, t) \in \Gamma^{\text {type }} \quad \text { (Neumann), } \\
& (\mathbf{v} u-\mathbf{D} \nabla u)(\mathbf{x}, t) \cdot \mathbf{n}=g_{3}^{\text {type }}(\mathbf{x}, t), \quad(\mathbf{x}, t) \in \Gamma^{\text {type }} \quad(\text { Robin }),
\end{aligned}
$$

where type $=I, O, N$ represents an inflow, outflow, or noflow boundary type.

Solutions to convection-dominated $(|\mathbf{v}| \gg|\mathbf{D}|)$ convection-diffusion problems exhibit physical features such as steep fluid fronts, current sheets, and shocks, which pose serious challenges to numerical methods for these problems. Standard finite difference or finite element methods produce either excessive nonphysical oscillations or extra numerical diffusion, which smears these physical features. The Eulerian-Lagrangian method developed in [5] (see also the references therein) is a characteristic FEM for these problems. This method naturally incorporates all types of boundary conditions in its formulation, is not subject 
to the severe restrictions imposed by the Courant-Friedrichs-Lewy (CFL) condition, and generates accurate numerical solutions even if large time steps are used. We briefly review this method in this section.

Let $0=t_{0}<t_{1}<\cdots<t_{n-1}<t_{n}<\cdots<t_{N}=T$ be a partition of $[0, T]$ with $\Delta t_{n}=t_{n}-t_{n-1}$. We multiply equation (3) by a test function $w(\mathbf{x}, t)$ that vanishes outside the space-time strip $\Omega \times\left(t_{n-1}, t_{n}\right]$ and is discontinuous in time at time $t_{n-1}$. Integration by parts leads to the following weak form

$$
\begin{aligned}
& \int_{\Omega} u\left(\mathbf{x}, t_{n}\right) w\left(\mathbf{x}, t_{n}\right) d \mathbf{x}+\int_{t_{n-1}}^{t_{n}} \int_{\Omega}(\mathbf{D} \nabla u) \cdot \nabla w d \mathbf{x} d t \\
& \quad+\int_{t_{n-1}}^{t_{n}} \int_{\partial \Omega}(\mathbf{v} u-\mathbf{D} \nabla u) \cdot \mathbf{n} w d S d t-\int_{t_{n-1}}^{t_{n}} \int_{\Omega} u\left(w_{t}+\mathbf{v} \cdot \nabla w\right) d \mathbf{x} d t \\
& =\int_{\Omega} u\left(\mathbf{x}, t_{n-1}\right) w\left(\mathbf{x}, t_{n-1}^{+}\right) d \mathbf{x}+\int_{t_{n-1}}^{t_{n}} \int_{\Omega}(f w)(\mathbf{x}, t) d \mathbf{x} d t
\end{aligned}
$$

where $d S$ is the differential element on $\partial \Omega$ and $w\left(\mathbf{x}, t_{n-1}^{+}\right):=\lim _{t \rightarrow t_{n-1}^{+}} w(\mathbf{x}, t)$ arises from the fact that $w(\mathbf{x}, t)$ is discontinuous in time at time $t_{n-1}$.

In the Eulerian-Lagrangian method, all test functions are chosen to satisfy the adjoint equation

$$
w_{t}+\mathbf{v} \cdot \nabla w=0
$$

Characteristic tracking is also an important part of the method. One solves the following initial value problem by numerical methods, e.g., the 2 nd order Runge-Kutta method, since an exact solution is usually unavailable.

$$
\left\{\begin{array}{l}
\frac{d \mathbf{y}}{d s}=\mathbf{v}(\mathbf{y}(s ; \mathbf{x}, t), s), \\
\left.\mathbf{y}(s ; \mathbf{x}, t)\right|_{s=t}=\mathbf{x} .
\end{array}\right.
$$

Let $\Gamma_{n}^{I}$ be the restriction of $\Gamma^{I}$ on $\left[t_{n-1}, t_{n}\right]$ and similarly for $\Gamma_{n}^{O}$. Variable time steps $\Delta t^{I}\left(\mathbf{x}, t_{n}\right), \Delta t^{O}(\mathbf{y}, t)$ are defined according to backward tracking [5]. Truncation of the diffusion and the source terms (the 2nd terms on the left and the right sides of (5)) leads to the following numerical scheme

$$
\begin{aligned}
\int_{\Omega} u & \left(\mathbf{x}, t_{n}\right) w\left(\mathbf{x}, t_{n}\right) d \mathbf{x}+\int_{\Omega} \Delta t^{I}\left(\mathbf{x}, t_{n}\right)(\mathbf{D} \nabla u \cdot \nabla w)\left(\mathbf{x}, t_{n}\right) d \mathbf{x} \\
& \left.+\int_{\Gamma_{n}^{O}} \Delta t^{O}(\mathbf{y}, t)(\mathbf{D} \nabla u) \cdot \nabla w\right)(\mathbf{y}, t)(\mathbf{v} \cdot \mathbf{n}) d S \\
& +\int_{\Gamma_{n}^{O}}(\mathbf{v} u-\mathbf{D} \nabla u) \cdot \mathbf{n} w(\mathbf{y}, t) d S+\int_{\Gamma_{n}^{I}}(\mathbf{v} u-\mathbf{D} \nabla u) \cdot \mathbf{n} w(\mathbf{y}, t) d S \\
= & \int_{\Omega} u\left(\mathbf{x}, t_{n-1}\right) w\left(\mathbf{x}, t_{n-1}^{+}\right) d \mathbf{x}+\int_{\Omega} \Delta t^{I}\left(\mathbf{x}, t_{n}\right) f\left(\mathbf{x}, t_{n}\right) w\left(\mathbf{x}, t_{n}\right) d \mathbf{x} \\
& +\int_{\Gamma_{n}^{O}} \Delta t^{O}(\mathbf{y}, t) f(\mathbf{y}, t) w(\mathbf{y}, t)(\mathbf{v} \cdot \mathbf{n}) d S .
\end{aligned}
$$

A finite element implementation seeks the trial function $U_{n}(\mathbf{x})$ from a conforming finite element space $\mathcal{S}_{h}$. Each test function $w(\mathbf{x}, t)$ needs to satisfy the adjoint 
equation (6) and also $w\left(\mathbf{x}, t_{n}\right) \in \mathcal{S}_{h}$. Then the discretized system has a banded symmetric positive definite coefficient matrix and the linear system can be solved by the conjugate gradient method.

\section{Numerical Experiments on the Resistivity Scales}

In this section, we perform numerical experiments on a test case that simulates the role of the magnetic field in a convecting plasma. The example was originally proposed in [7] and recently studied in [8]. In this paper, the Eulerian-Lagrangian method discussed in the previous section is used as a unified numerical method for the two dimensional magnetic potential equation (2). By "unified", we mean that the solver can be applied to the equation with or without the resistivity term. Lagrangian $\mathcal{P}_{1}$ elements are used on a $128 \times 128 \times 2$ structured triangular mesh (a rectangle is divided into two triangles). The second order Gaussian quadrature is employed for all triangle elements. The solver uses a large time step $\Delta t=0.1$. The 2 nd order Runge-Kutta method with 16 micro steps is used for tracking characteristics. We test the example with four different values for resistivity $\left(\varepsilon=0,5 \times 10^{-7}, 5 \times 10^{-5}, 2 \times 10^{-2}\right)$ and observe the changes in the magnetic field energy and topology.

The spatial domain is $\Omega=[-0.5,0.5]^{2}$. The velocity field is prescribed as

$$
\mathbf{v}(x, y)=(-\sin (2 \pi x) \cos (2 \pi y), \cos (2 \pi x) \sin (2 \pi y)) .
$$

All four sides of the domain are noflow boundaries. The boundary conditions are

$$
\left.\left(A_{3}\right)\right|_{x=-0.5}=1,\left.\quad\left(A_{3}\right)\right|_{x=0.5}=0,\left.\quad \frac{\partial A_{3}}{\partial n}\right|_{y=-0.5}=\left.\frac{\partial A_{3}}{\partial n}\right|_{y=0.5}=0
$$

The initial condition shown in Figure 1 is $\left.\left(A_{3}\right)\right|_{t=0}=0.5-x$, which corresponds to an initial (horizontally) uniform magnetic field.
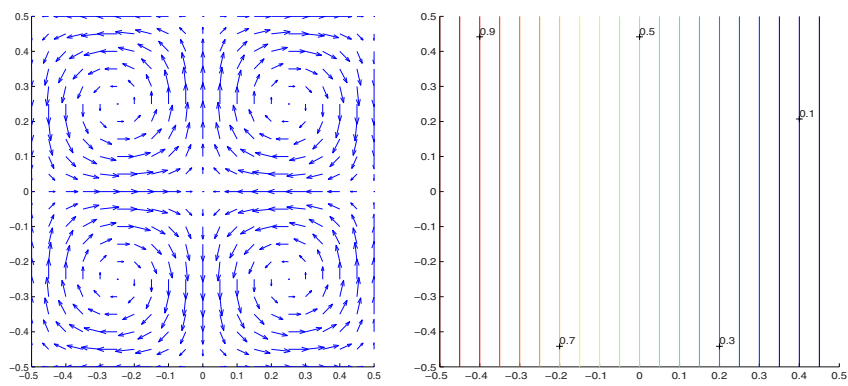

Fig. 1. (Left): the velocity field; (Right): the initial uniform magnetic potential, contour line values from 0 to 1 with an increment 0.05 
This model problem exhibits different scales of resistivity. The convection and diffusion processes behave at disparate scales. But the characteristic FEM discussed in Section 2 is an efficient solver for this type of problems. Accordingly, there are two time scales in the numerical simulation. The macro time step for the numerical scheme (8) is related to diffusion and could be relatively large. It is not subject to the CFL condition. But the convection happens at a much faster pace. Small micro time steps are used for solving equations (6) and (7), so that steep fluid fronts can be accurately resolved.

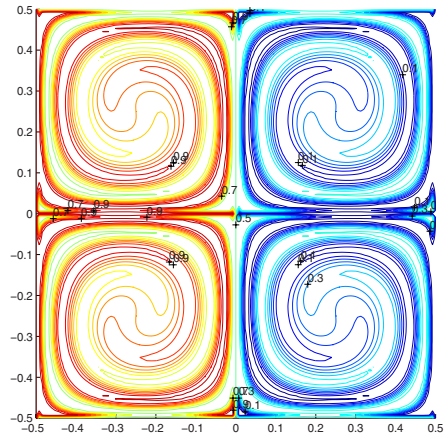

(a)

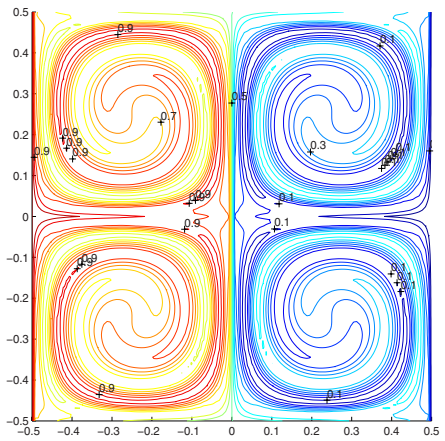

(c)

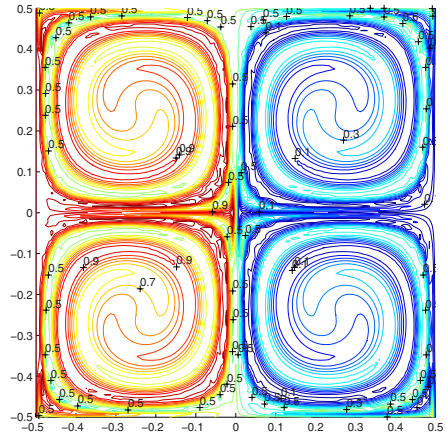

(b)

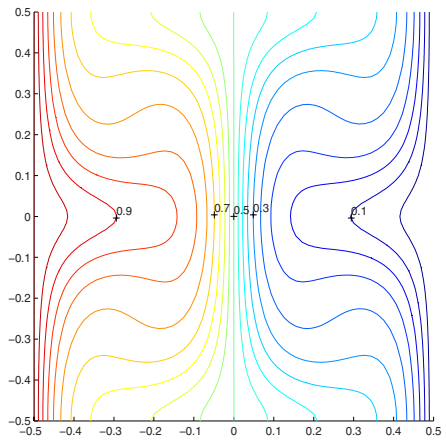

(d)

Fig. 2. Topology of the magnetic field at the same time $T=3$ with different resistivity: (a) $\varepsilon=0$; (b) $\varepsilon=5 \times 10^{-7}$; (c) $\varepsilon=5 \times 10^{-5}$; (d) $\varepsilon=2 \times 10^{-2}$

Note that contours of the magnetic potential are actually magnetic field lines. When resistivity disappears, the magnetic equation becomes a pure convection equation, the magnetic field is "frozen", In other words, a thin rope-like volume of fluid surrounding a magnetic field line will continue to lie along the line, even though it might be twisted and distorted [2]. The topology of the magnetic field does not change. However, the geometry of the magnetic field changes due to the convection, and as a result, the magnetic energy increase. This can be observed in the left subplot of Figure 3. It is also known from Figure2(a) that all magnetic field lines are confined to each of the four convection cells. 

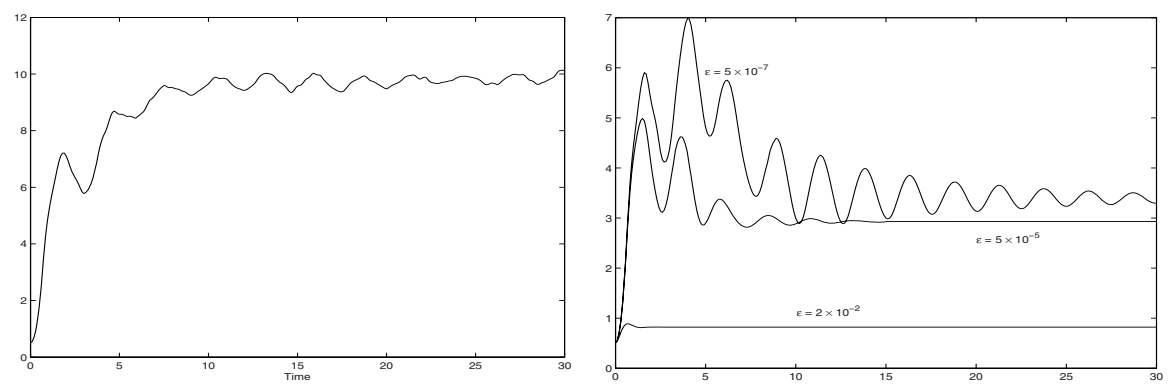

Fig. 3. Evolution of the magnetic field energy over the time period $[0, T]=[0,30]$. (Left): $\varepsilon=0$; (Right): $\varepsilon=5 \times 10^{-7}, 5 \times 10^{-5}, 2 \times 10^{-2}$ (top, middle, bottom, respectively).

Table 2. Maximal magnetic field strength at various time moments

\begin{tabular}{||c||c|c|c|c||}
\hline & $\varepsilon=0$ & $\varepsilon=5 \times 10^{-7}$ & $\varepsilon=5 \times 10^{-5}$ & $\varepsilon=2 \times 10^{-2}$ \\
\hline$T=0.1$ & 1.867 & 1.867 & 1.865 & 1.705 \\
$T=1$ & 60.837 & 49.917 & 40.281 & 4.839 \\
$T=5$ & 66.807 & 66.940 & 31.122 & 4.671 \\
$T=10$ & 64.000 & 65.251 & 33.406 & 4.671 \\
$T=30$ & 64.000 & 59.101 & 33.340 & 4.671 \\
\hline
\end{tabular}

When resistivity is present, the magnetic field diffuses through the fluid in a manner similar to the diffusion of heat through a solid. The change of the magnetic flux on a closed curve due to the resistivity can produce a nonzero component in the electric field that is parallel to the magnetic field, but this does not imply magnetic reconnection will necessarily happen [2]. As shown in Figure 2, a resistivity of $\varepsilon=2 \times 10^{-2}$ is too strong, no magnetic reconnection can happen. However, for $\varepsilon=5 \times 10^{-5}$, magnetic reconnection happens around $T=3$. Magnetic field lines from different magnetic domains are spliced to one another, changing the overall topology of the magnetic field. An X-type conjunction can be observed in Figure 2(c).

In real plasma physics, an X-type neutral line is believed to form between the Earth's magnetic field and a solar wind [1]. The evolution of the coronal magnetic field due to magnetic reconnection was investigated in [6] .

Figure 3 shows the magnetic field energy evolution for all four cases. For $\varepsilon=2 \times 10^{-2}$, the strong resistivity quickly brings the magnetic field to its steady state. For $\varepsilon=5 \times 10^{-5}$, the time period for the magnetic field to reach its equilibrium is relatively longer. In general, this is related to the Alfvén time scale of a plasma 2]. All four cases exhibit an energy spike in the early stage, due to the energy infusion from the initial convection-dominance. Table 2 lists the maximal strength of the magnetic fields at several time moments. These results are consistent with those about energy evolution. 


\section{Concluding Remarks}

Other examples on applying the characteristic FEM to resolve the kinematics of 2-dim resistive MHD flows can be found in 4, which contains also a comparison of the numerical solutions of the time-dependent convection-diffusion equation for magnetic induction and the steady-state convection-diffusion equation describing the equilibrium of a magnetic field. While 4] examines the role of velocity fields, this paper focuses on the role of the scale of resistivity.

The divergence-free property is an important physical property that should be respected by numerical methods. Another approach is to use locally divergencefree (LDF) finite elements, especially for the three dimensional magnetic induction equation. Combining LDF finite elements and the discontinuous Galerkin method to solve the ideal MHD equations has been reported in [3. We are developing numerical methods for the resistive magnetic induction equation based on combination of characteristic tracking and discontinuous or nonconforming LDF finite elements. The results will be reported elsewhere.

This paper focuses on the role of resistivity in the kinematics of MHD, This is a one-way coupling. But MHD is a coupled system about the interaction between fluid motion and electromagnetism. Numerical methods for the fully coupled problems in resistive MHD are the topics of our ongoing research projects.

\section{References}

1. Dungey, J.W.: Interplanetary Magnetic Field and the Auroral Zones. Phys. Rev. Lett. 6 (1961) 47-48

2. Gurnett, D.A., Bhattacharjee, A.: Introduction to Plasma Physics with Space and Laboratory Applications, Cambridge University Press. 2005

3. Li, F., Shu, C.W.: Locally Divergence-free Discontinuous Galerkin Methods for MHD equations. J. Sci. Comput. 22/23 (2005) 413-442

4. Liu, J., Tavener, S., and Chen, H.: ELLAM for Resolving the Kinematics of Two Dimensional Resistive Magnetohydrodynamic Flows. Preprint.

5. Wang, H., Dahle, H.K., Ewing, R.E., Espedal, M.S., Sharpley, R.C., Man, S.: An ELLAM Scheme for Advection-Diffusion Equations in Two Dimensions. SIAM J. Sci. Comput. 20 (1999) 2160-2194

6. Wu, S.T., Wang, A.H., Plunkett, S.P., Michels, D.J.: Evolution of Gloabl-scale Coronal Magnetic Field due to Magnetic Reconnection: The Formulation of the Observed Blob Motion in the Coronal Steamer Belt. Astro. Phys. J. 545 (2000) 1101-1115

7. Weiss, N.O.: The Expulsion of Magnetic Flux by Eddies. Proc. Roy. Soc. A. 293 (1966) 310-328

8. Zegeling, P.A.: On Resistive MHD Models with Adaptive Moving Meshes. J. Sci. Comput. 24 (2005) 263-284 